\title{
Principais instrumentos para avaliação da disfunção temporomandibular, parte I: índices e questionários; uma contribuição para a prática clínica e de pesquisa Main instruments for assessing temporomandibular disorders, part I: indices and
questionnaires; a contribution to clinicians and researchers
}

Thaís Cristina Chaves', Anamaria Siriani de Oliveira2, Débora Bevilaqua Grossi ${ }^{3}$

1 Fisioterapeuta; Ms.

2 Fisioterapeuta; Profa. Dra. do Depto. de Biomecânica, Medicina e Reabilitação do Aparelho Locomotor, FMRP/ USP (Faculdade de Medicina de Ribeirão Preto da Universidade de São Paulo, Ribeirão Preto, SP)

3 Fisioterapeuta; Profa. Associada do Depto. de Biomecânica, Medicina e Reabilitação do Aparelho Locomotor, FMRP/USP

ENDEREÇO PARA

CORRESPONDÊNCIA

Débora Bevilaqua Grossi

Depto. de Biomecânica - RAL

FMRP/USP Campus

Universitário

14049-900 Ribeirão Preto SP e-mail: deborabg@fmrp.usp.br

APRESENTAÇÃO set. 2006

ACEITO PARA PUBLICAÇÃO ago. 2007
Resumo: Na literatura especializada, encontram-se variados instrumentos para avaliação da disfunção temporomandibular (DTM), sob a forma de índices, questionários, protocolos, escalas de avaliação e critérios de diagnóstico. Este estudo, dividido em duas partes, visou caracterizar os principais instrumentos de avaliação da DTM disponíveis na literatura, para auxiliar o clínico e o pesquisador na correta escolha da ferramenta apropriada para contemplar seus objetivos clínicos ou científicos. Nesta parte I são apresentados dois índices clínicos e três questionários (anamnésicos e funcionais); na parte II, um questionário funcional e dois conjuntos de critérios diagnósticos. Os índices são ferramentas que organizam a avaliação de sinais e sintomas, pela obtenção de pontuações. Os questionários são melhor aplicados para traçar perfis populacionais em estudos epidemiológicos. Para avaliação dos eventuais impactos da DTM nas atividades de vida diária, os questionários funcionais são mais adequados. Finalmente, há poucos conjuntos de critérios sistematizados para obter o diagnóstico da disfunção. A utilização de uma ou outra ferramenta depende de sua aplicabilidade e dos objetivos do profissional que a irá utilizar.

Descritores: Avaliação; Índice de gravidade de doença; Questionários; Transtornos da articulação temporomandibular

ABSTRACT: Several instruments for assessing temporomandibular disorders (TMD) are available in literature, such as indices, questionnaires, protocols, scales, and diagnostic criteria. The purpose of this study, divided into two parts, was to characterise main tools available for TMD evaluation, so as to offer both researchers and clinicians guiding information on instrument selection according to their clinical or research needs. Two clinical indices and three (anamnestic and functional) questionnaires are presented here in part I; and, in part II, one functional questionnaire and two diagnostic criteria. Indices consist of organised forms for assigning scores to signs and symptoms thus obtaining severity degrees. Questionnaires are better employed for epidemiological purposes. Functional questionnaires are better used to estimate impact of TMD on daily life activities. There are few specific sets of systematised diagnostic criteria available for diagnosing TMD. The use of either tool depends on its applicability and on the user's purposes.

KEY wORDs: Evaluation; Questionnaires; Severity of illness index; Temporomandibular joint disorders 


\section{INTRODUÇÃO}

Disfunção temporomandibular (DTM) é um termo que se aplica às alterações funcionais relativas à articulação temporomandibular (ATM) e estruturas mastigatórias associadas ${ }^{1}$. DTM refere-se a um aglomerado de desordens caracterizadas por ruídos articulares, limitações na amplitude de movimento ou desvios durante a função mandibular, que são considerados sinais de DTM, e dor pré-auricular, dor na ATM ou nos músculos mastigatórios, caracterizados como sintomas ${ }^{2}$.

A etiologia da DTM é multifatorial ${ }^{3}$. Traumas da mandíbula ou ATM, má oclusão e interferências oclusais, alterações nos músculos mastigatórios, microtraumas provocados por hábitos parafuncionais contínuos ${ }^{1}$, condições reumáticas, estresse emocional, ansiedade e anormalidades posturais ${ }^{4-8}$ podem estar relacionados com o desenvolvimento de DTM. Considerando-se que esta é uma condição caracterizada por um conjunto de sinais e sintomas ${ }^{2,9,-11}$, estes devem ser organizados de maneira clara, padronizada e operacional para avaliação e classificação correta da DTM, tanto funcional quanto diagnóstica.

$\mathrm{Na}$ literatura, pode-se verificar a existência de diferentes instrumentos para avaliação de DTM organizados sob diversas formas: questionários ${ }^{12}$, índices anamnésicos ${ }^{13,14}$ e clínicos ${ }^{13,15,16}$ e critérios de diagnóstico ${ }^{17,18}$. Cada uma dessas ferramentas apresenta vantagens, desvantagens e limitações, bem como aplicabilidades distintas. Assim, tanto o clínico quanto o pesquisador precisam estar cientes dos dados que podem ser obtidos com a aplicação de cada instrumento, para empregá-lo adequadamente.

Este artigo tem por objetivo caracterizar os principais instrumentos de avaliação da DTM existentes na literatura e fornecer esclarecimentos quanto à natureza dos dados obtidos com seu emprego, auxiliando o clínico e o pesquisador na correta escolha da ferramenta apropriada para contemplar seus objetivos clínicos ou científicos. Nesta parte I são apresentados os índices e questionários e, na parte II, um questionário funcional e os critérios diagnósticos.

\section{METODOLOGIA}

Os instrumentos aqui apresentados resultam de uma pesquisa na base de dados PubMed de artigos recentes (publicados entre 2000 e 2007) mediante o descritor temporomandibular joint disorders associado a assessment, index e clinical diagnosis. Foram retidos os artigos referentes às ferramentas mais citadas e que contivessem a íntegra do instrumento proposto.

\section{ÍNDICES}

Os índices encontrados na literatura são classificados como anamnési$\cos ^{13,14}$ ou clínicos ${ }^{13,15,16}$. Em geral, caracterizam-se pelo agrupamento operacional de conjuntos de sinais e ou sintomas sob a forma de itens e subitens, para os quais são predeterminadas pontuações ou scores que, ao final, são somados e permitem classificar os voluntários em subcategorias funcionais ou de severidade.

\section{Índice de disfunção clínica craniomandibular (IDCCM)}

Helkimo $^{13}$ foi um dos pioneiros a desenvolver um índice para avaliação da DTM. Os estudos iniciais que conduziu visavam uma investigação epidemiológica da função e disfunção do sistema mastigatório na Finlândia. O Índice de Disfunção Clínica Craniomandibular-IDCCM(Clinical Dysfunction Index, ou Índice de Helkimo) tem por objetivo classificar os voluntários em categorias de severidade de sinais clínicos de DTM. É subdividido em cinco itens: limitação na amplitude de movimento mandibular, limitação na função da ATM, dor muscular, dor na ATM e dor no movimento mandibular. Para cada item são possíveis três pontuações, dependendo da avaliação clínica: 0, 1 e 5 (Quadro 1). Ao final, a somatória das pontuações de cada item permite classificar os voluntários em quatro categorias: sem sintomas de DTM (0 ponto), sintomas leves ( 1 a 4 pontos), moderados (5 a 9 pontos) e severos (10 a 25 pontos). Os dados necessários para preencher o item " $a$ " do índice clínico devem ser obtidos pelo Índice de Mobilidade Mandibular (IMM) (Quadro 1).

O índice de Helkimo é um dos primeiros índices preconizados na literatura e, apesar de algumas limitações, tem sido empregado na literatura $^{19-22}$. No entanto, não fornece classificação diagnóstica, apenas avaliação de severidade de sinais e sintomas, opondo-se às tendências atuais na área de DTM, em que se preconiza a abordagem diagnóstica. Além disso, as propriedades psicométricas desse índice ainda não foram verificadas ${ }^{23}$ e, dessa forma, sua aplicabilidade clínica em distinguir pacientes de nãopacientes ainda não foi evidenciada. Outra limitação do índice é seu sistema de pontuação: apenas o voluntário que apresentar ausência total de sinais e sintomas de DTM é classificado como "assintomático"; valores de pontuação entre um e 25 pontos já classificam o voluntário como portador de algum grau de severidade de DTM. Dessa forma, se um voluntário apresentar apenas estalidos durante o movimento mandibular, de acordo com o índice de Helkimo já apresentará algum grau de severidade de DTM. Tendo em vista que a prevalência de sons articulares na população geral é alta, em torno de $34 \%{ }^{24}$ e nem sempre são indicativos clínicos de disfunção da $\mathrm{ATM}^{17}$, a possibilidade de classificar um voluntário saudável em algum grau de severidade do índice demonstra uma falha importante no conjunto de sinais e sintomas organizados sob seu sistema de pontuação.

\section{ÍndiceCraniomandibular(ICM) ou Índice Temporomandibular(ITM)}

Fricton e Schiffman ${ }^{15,25}$ propuseram, para avaliação de DTM, o Índice 
Quadro 1 Índice de Helkimo ${ }^{13}$ ou de Disfunção Clínica Craniomandibular (IDCCM) com Índice de Mobilidade Mandibular (IMM) [Tradução não-oficial]

a) Índice de amplitude de movimento (ver abaixo IMM)

Variação normal de movimento

Movimento levemente prejudicado

Movimento severamente prejudicado

Pontos

0

5

b) Dor ao movimento da mandíbula

Nenhuma dor ao movimento

Dor durante um movimento

Dor durante dois ou mais movimentos

c) Dor na ATM

Nenhuma dor à palpação

Dor à palpação lateral

Dor à palpação posterior

d) Alterações na função da ATM (movimento de abertura ou fechamento)

Movimento suave, sem ruído na ATM, com desvio $\leq 2 \mathrm{~mm}$

Ruídos na ATM em uma ou ambas as articulações e/ou desvio $\geq 2 \mathrm{~mm}$

Travamento e/ou luxação da ATM

e) Dor muscular

Nenhuma sensibilidade à palpação nos músculos mastigatórios

Sensibilidade à palpação em uma a três áreas

Sensibilidade à palpação em quatro ou mais áreas

Soma: $\mathrm{a}+\mathrm{b}+\mathrm{c}+\mathrm{d}+\mathrm{e}$

0

1

5

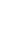

0

1

5

0

1

5

0

1

5

\begin{tabular}{|c|c|c|c|}
\hline \multirow[b]{2}{*}{ Valor total } & \\
\hline & Intervalo & Índice de disfunção & Classificação da disfunção \\
\hline & 0 & Índice 0 & Nenhuma disfunção \\
\hline & $1-4$ & Índice 1 & Disfunção suave \\
\hline & $5-9$ & Índice 2 & Disfunção moderada \\
\hline & $10-13$ & Índice 3 & Disfunção severa \\
\hline & $15-17$ & Índice 4 & Disfunção severa \\
\hline & $20-25$ & Índice 5 & Disfunção severa \\
\hline
\end{tabular}

Índice de mobilidade mandibular - IMM

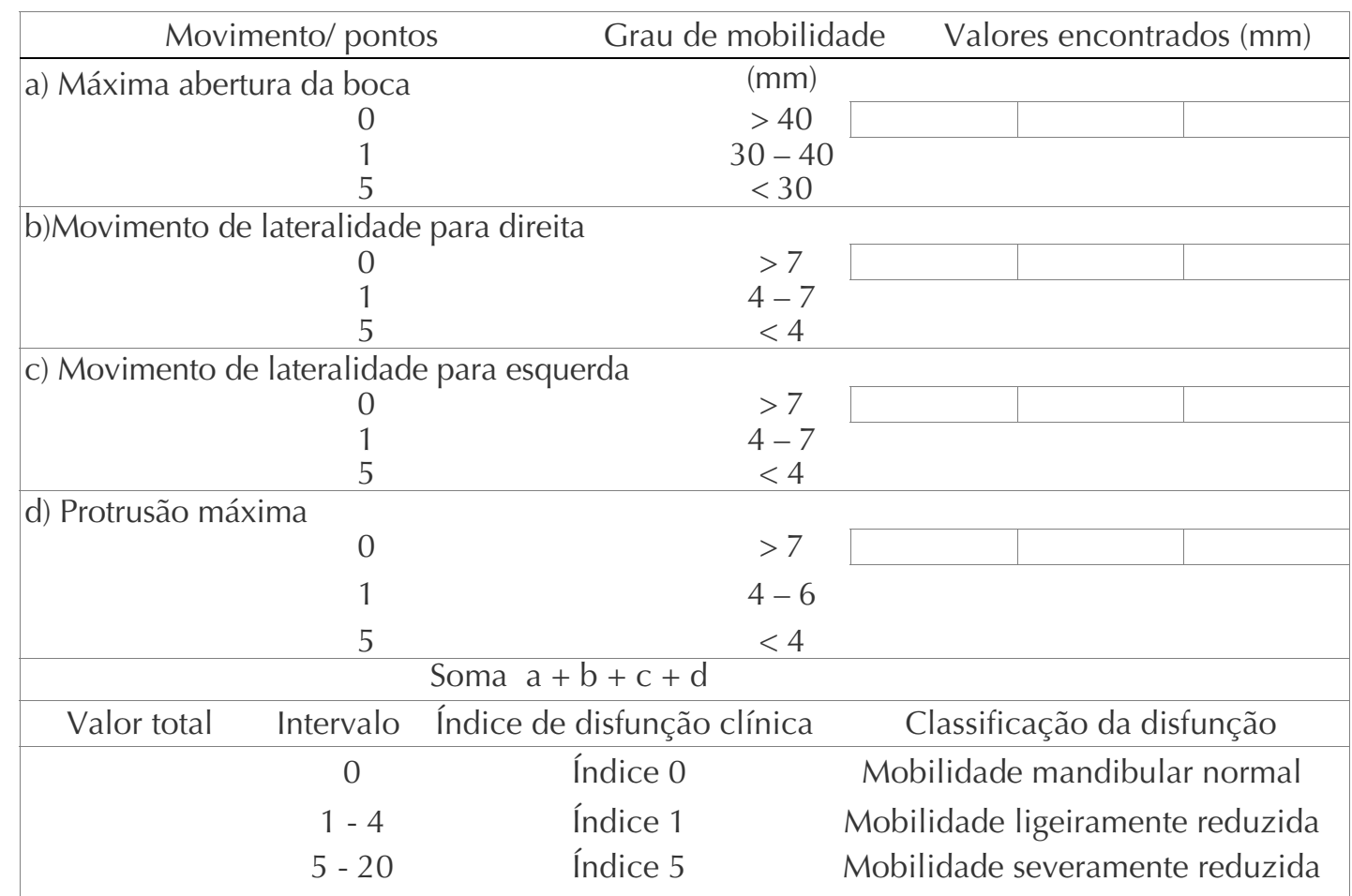


Quadro 2 Índice Temporomandibular de Fricton e Shiffman ${ }^{15,25}$ [Tradução não-oficial do apresentado por Pehling et al. ${ }^{26}$ ] 1 Índice funcional

Amplitude de movimento

Dor durante

Máxima abertura da boca ativa sem dor $(\geq 40 \mathrm{~mm})$ $\mathrm{mm}(0)(1)$ movimentação

Máxima abertura da boca ativa $(\geq 40 \mathrm{~mm})$ $\mathrm{mm}(0)(1)$

(0) (1)

Máxima abertura passiva da boca $(\geq 40 \mathrm{~mm})$

Desvio lateral direito $(\geq 7 \mathrm{~mm})$

Desvio lateral esquerdo $(\geq 7 \mathrm{~mm})$

Protrusão $(\geq 7 \mathrm{~mm})$

Superposição vertical dos incisivos

$\pm \quad \mathrm{mm}$

Padrão de abertura (marque apenas uma linha nesta seção)

Sem presença de desvio da linha média

Desvio com retorno para a linha média

(0)

(1)

Deflexão

Outro

Índice funcional: Total de respostas positivas

Lado esquerdo

2 Índice muscular: dor à palpação de áreas musculares Lado direito
(0) (1) Temporal anterior

(0) (1) Temporal médio

(0) (1) Temporal posterior

(0) (1) Origem do masseter

(0) (1) Ventre do masseter

(0) (1) Inserção do masseter

(0) (1) Região posterior da mandíbula

(0) (1) Região submandibular

(0) (1) Área do pterigóideo lateral

(0) (1) Tendão do temporal

Índice muscular $=$ Total de respostas positivas

Lado esquerdo

Palpação da ATM: Lado direito

Pólo lateral

(0) (1) Pólo lateral

(0) (1) Região posterior

(0) (1)

Região posterior

(0)..........................

Pontuação dos sons articulares da ATM: conte apenas um positivo por lado para as seções A e B Lado direito

Lado esquerdo

Estalido reprodutível durante abertura

Estalido reprodutível durante fechamento

(0) (1) Estalido reprodutível durante abertura

(0) (1)

(0) (1) Estalido reprodutível durante fechamento

(0) (1)

(0) (1) Estalido recíproco reprodutível

(0) (1) Estalido reproduzível na lateralização

(0) (1)

Estalido reproduzível na lateralização

Estalido reprodutível na protrusão

(0) (1) Estalido reprodutível na protrusão

(0) (1)

(0) (1)

(0) (1) Estalido não-reprodutível*

(0) (1)

*Estalidos não-reprodutíveis em qualquer movimento mandibular não são válidos para pontuação Lado direito

Crepitação áspera

Crepitação fina

(0) (1) Crepitação áspera

(0) (1)

(0) (1) Crepitação fina Índice articular: Total de respostas positivas _ $/ 8=$

ITM: Índice funcional + Índice muscular + Índice articular / 3 = 
Craniomandibular, que foi posteriormente revisado e renomeado Índice Temporomandibular, tendo suas propriedades psicométricas testadas ${ }^{16,26}$. Esse índice é subdividido em três: Índice Funcional, Índice Muscular e Índice Articular (Quadro 2). Em cada subíndice são listados itens específicos relativos aos sinais clínicos de DTM, para os quais é possível atribuir valor zero, na ausência do sinal clínico, ou valor 1 , na presença do sinal clínico. As somas das respostas positivas para cada subíndice são, ao final, divididas pelo número total de itens, obtendose o grau de comprometimento em cada nível. Dessa forma, pode-se classificar os voluntários em porcentagens de comprometimento nos níveis funcional, muscular e articular. No entanto, diferentemente dos índices de Helkimo $^{13}$ e de Fonseca et al. ${ }^{14}$, não é possível, com esse índice, classificar os voluntários em categorias de severidade de sinais clínicos e sintomas. A validade de critério e de construto ${ }^{26}$ já foi testada para esse instrumento, bem como a confiabilidade intra e interexaminadores ${ }^{16}$.

Uma das justificativas alegadas pelos autores para esse índice é que forneceria, em uma única avaliação clínica, a classificação diagnóstica de DTM e a classificação de severidade da disfunção. Entretanto, os autores não fornecem um sistema de pontuação para a classificação em níveis de severidade. Tampouco são oferecidos critérios operacionais para obtenção do diagnóstico com base nos dados clínicos obtidos, portanto esse índice não permite tal classificação. Porém, apesar de os autores não definirem valores de corte para diferenciar pacientes de não-pacientes, Pehling et al. ${ }^{16}$ demonstraram que, usando as pontuações obtidas pelo ITM, é possível distinguir pacientes diagnosticados nos subgrupos do RDC/TMD (Critérios diagnósticos para DTM, Research Diagnostic Criteria for Temporomandibular Disorders - ver parte II, neste número da revista).
QUESTIONÁRIOS

Os questionários são amplamente utilizados na literatura para avaliação dos sintomas de DTM ${ }^{14,27-32}$ e podem ser utilizados de diferentes formas: em entrevista pessoal, com auxílio de um entrevistador ou não (auto-administráveis) e por telefone.

Os questionários específicos para DTM são ferramentas adequadas para estudos epidemiológicos ou populacionais, nos quais o objetivo é traçar perfis populacionais dos sintomas de DTM. Assim, a avaliação obtida pode ser útil para triar pacientes em potencial para pesquisas e para avaliação inicial na clínica ${ }^{30}$. Apesar de úteis, os questionários devem ser interpretados com cautela, uma vez que a presença de sinais e sintomas de DTM não necessariamente é indicativa da presença de DTM, devido à baixa especificidade dessas ferramentas, quando incorretamete utilizadas como instrumentos de diagnóstico ${ }^{33}$. Gerstner et al. ${ }^{30}$ chamam a atenção para a necessidade de se associar à avaliação de sintomas uma avaliação clínica mais direcionada.

\section{Questionário e Índice Anamnésico de Fonseca}

O Questionário anamnésico de Fonseca et al. ${ }^{14}$ foi elaborado nos moldes do Índice anamnésico de Helkimo $^{13}$, e é um dos poucos instrumentos disponíveis em língua portuguesa para caracterizar a severidade dos sintomas de DTM. Foi previamente testado em pacientes com DTM ${ }^{14}$ e demonstrou uma correlação de 95\% com o índice clínico de Helkimo ${ }^{13}$.

Para cada uma das questões do questionário de Fonseca são possíveis três respostas (sim, não e às vezes) para as quais são preestabelecidas três pontuações (10, 0 e 5, respectivamente) (Quadro 3). Com a somatória dos pontos atribuídos obtém-se um índice anamnésico que permite classificar os voluntários em categorias de severi- dade de sintomas: sem DTM (0 a 15 pontos), DTM leve (20 a 45 pontos), DTM moderada (50 a 65) e DTM severa (70 a 100 pontos).

Alguns estudos brasileiros têm utilizado o índice de Fonseca para classificação dos pacientes quanto aos sintomas de DTM ${ }^{8,34-36}$. A simplicidade desse índice favorece seu uso em estudos epidemiológicos populacionais. No entanto, ainda não foi completamente validado e também não oferece classificação diagnóstica de DTM; os dados obtidos com esse índice são pois restritos à classificação de severidade de sinais e sintomas de DTM. Outra limitação é seu sistema de pontuação, uma vez que se três respostas afirmativas forem atribuídas às questões sobre relato de dor de cabeça, dor cervical e percepção de tensão emocional, o voluntário será classificado como portador de DTM leve. No entanto, esses mesmos sintomas podem ocorrer de maneira isolada, sem que exista qualquer associação com a DTM.

\section{Questionário da Academia Americana de Dor Orofacial}

A American Academy of Orofacial Pain recomenda um questionário ${ }^{12}$ para triagem inicial de potenciais pacientes com dores orofaciais, inclusive DTM. Esse questionário apresenta dez questões especificas relacionadas à DTM (Quadro 4). Entretanto, o próprio autor recomenda que a triagem por questionário deva ser associada à coleta de dados pela história clínica e exame clínico.

Manfredi et al. ${ }^{32}$ aplicaram esse questionário para triagem de dor orofacial em 46 pacientes com DTM e verificaram uma sensibilidade de $85,37 \%$ e especificidade de $80 \%$ para pacientes com DTM miogênica. No entanto, para disfunções articulares foram verificados baixos níveis de sensibilidade e especificidade ${ }^{32}$. Os autores concluem que o questionário é útil para uma pré-triagem dos pacientes e, como mencionado, não permite a obtenção de diagnóstico. 
Quadro 3 Questionário anamnésico de Fonseca ${ }^{14}$ (com o qual se obtém o Índice Anamnésico de Fonseca)

\begin{tabular}{|c|c|c|}
\hline \multicolumn{3}{|c|}{ Pergunta } \\
\hline \multicolumn{3}{|c|}{ Sente dificuldade para abrir a boca? } \\
\hline \multicolumn{3}{|c|}{ Você sente dificuldades para movimentar sua mandíbula para os lados? } \\
\hline \multicolumn{3}{|c|}{ Tem cansaço/dor muscular quando mastiga? } \\
\hline \multicolumn{3}{|c|}{ Sente dores de cabeça com freqüência? } \\
\hline \multicolumn{3}{|c|}{ Sente dor na nuca ou torcicolo? } \\
\hline \multicolumn{3}{|c|}{ Tem dor de ouvido ou na região das articulações (ATMs)? } \\
\hline \multicolumn{3}{|c|}{ Já notou se tem ruídos na ATM quando mastiga ou quando abre a boca? } \\
\hline \multicolumn{3}{|c|}{$\begin{array}{l}\text { Você já observou se tem algum hábito como apertar e/ou ranger os } \\
\text { dentes (mascar chiclete, morder o lápis ou lábios, roer a unha)? }\end{array}$} \\
\hline \multicolumn{3}{|c|}{ Sente que seus dentes não se articulam bem? } \\
\hline \multicolumn{3}{|c|}{ Você se considera uma pessoa tensa ou nervosa? } \\
\hline \multirow{2}{*}{$\begin{array}{l}\text { Obtenção } \\
\text { do índice: }\end{array}$} & Índice anamnésico & Grau de acometimento \\
\hline & $0-15$ & Sem DTM \\
\hline \multirow{3}{*}{$\begin{array}{l}\text { Soma dos pontos } \\
\text { atribuídos acima }\end{array}$} & $20-40$ & DTM leve \\
\hline & $45-65$ & DTM moderada \\
\hline & $70-100$ & DTM severa \\
\hline
\end{tabular}

Quadro 4 Questionário para avaliação de disfunção temporomandibular recomendado pela Academia Americana de Dor Orofacial $^{12}$ [Tradução oficial]

\begin{tabular}{|l|l|}
\hline Pergunta & Sim Não \\
\hline Você tem dificuldade, dor ou ambas ao abrir a boca, por exemplo, ao bocejar? \\
Sua mandíbula fica "presa", "travada" ou sai do lugar? \\
Você tem dificuldade, dor ou ambas ao mastigar, falar ou usar seus maxilares? \\
Você percebe ruídos na articulação de seus maxilares? \\
Seus maxilares ficam rígidos, apertados ou cansados com regularidade? \\
Você tem dor nas orelhas ou em volta delas, nas têmporas e bochechas? \\
Você tem cefaléia, dor no pescoço ou nos dentes com freqüência? \\
Você sofreu algum trauma recente na cabeça, pescoço ou maxilares? \\
Você percebeu alguma alteração recente na sua mordida? \\
Você fez tratamento recente para um problema não-explicado na articulação mandibular?
\end{tabular}

Questionário e Índice de Limitação Funcional Mandibular - MFIO

O Mandibular Function Impairment Questionnaire (MFIQ)29 ou Questionário de Limitação Funcional Mandibular, tal como o de Fonseca et al. ${ }^{14}$, tem um sistema de pontuação que o caracteriza como índice, possibilitando classificar os voluntários em categorias de severidade de limitação funcional relacionada à DTM. A valida- de de construto do questionário foi testada em uma amostra de 95 pacientes $^{29}$.

O questionário apresenta 17 questões para as quais são possíveis cinco respostas, com valores variando de 0 a 4 (Quadro 5). A pontuação total é obtida somando-se os valores das respostas a cada questão, mas a graduação não é linear. Os autores propuseram uma ponderação em que a pontuação é dividida pelo número de itens respondidos multiplicado por quatro (que é o valor máximo da resposta a cada questão); e o coeficiente assim obtido ainda deve ser cotejado a determinadas condições do conjunto de respostas, para se obter o grau de acometimento e a categoria de severidade. As regras para obtenção da graduação de severidade estão no Quadro 5.

Bom nível de consistência interna foi obtido para esse questionárioíndice ${ }^{29}$. Entretanto, os próprios autores sugerem que estudos adicionais devam ser conduzidos em diferentes amostras de pacientes com DTM; também, outras etapas do processo de validação ainda precisam ser conduzi- 
Quadro 5 Questionário e Índice de Limitação Funcional Mandibular (MFIQ) ${ }^{29}$ [Tradução não-oficial]

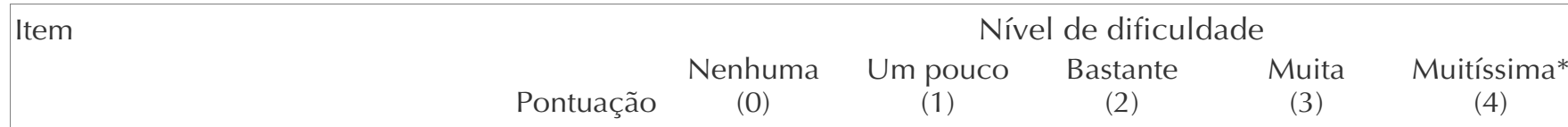

Com relação a queixas de dores na mandíbula, quanto de dificuldade você apresenta para realizar as seguintes atividades:

1 Atividades sociais

2 Falar

3 Dar uma boa mordida

4 Mastigar comida dura

5 Mastigar comida mole

6 Trabalhar ou realizar atividades de vida diária

7 Beber

8 Rir

9 Mastigar comida dura

10 Bocejar

11 Beijar

\begin{tabular}{|l|l|l|l|l|}
\hline & & & \\
\hline & & & & \\
\hline & & & & \\
\hline & & & & \\
\hline & & & & \\
\hline & & & & \\
\hline & & & & \\
\hline & & & & \\
\hline & & & & \\
\hline
\end{tabular}

Comer inclui morder, mastigar e deglutir. Quanto de dificuldade você tem para comer os seguintes alimentos:

1 Uma bolacha dura

2 Um bife

3 Uma cenoura crua

4 Um pão francês

5 Amendoim

6 Uma maçã

Soma das pontuações $\mathrm{S}=$

\begin{tabular}{|l|l|l|l|l|}
\hline & & & & \\
\hline & & & & \\
\hline & & & & \\
\hline & & & & \\
\hline & & & & \\
\hline
\end{tabular}

Cálculo do índice: $\mathrm{C}=\mathrm{S} / \mathrm{N} .4$, onde $\mathrm{S}=$ soma das pontuações obtidas e $\mathrm{N}=$ número de itens respondidos (divida a soma $\mathrm{S}$ encontrada pelo número de itens respondidos vezes 4)

Para chegar ao grau de acometimento funcional, calcule C e siga as regras da $1 \underline{a}$ coluna:

\begin{tabular}{|lcc|}
\hline \multicolumn{1}{|c|}{ Regras $(\mathrm{R}=$ resposta/s) } & Faixas de variação do índice C & $\begin{array}{c}\text { Grau de acometimento } \\
\text { funcional }\end{array}$ \\
\hline Todas as $\mathrm{R}$ com pontuação $<2$ & $\mathrm{C} \leq 0,3$ & 0 \\
Pelo menos uma $\mathrm{R} \geq 2$ & $\mathrm{C} \leq 0,3$ & 1 \\
Todas as $\mathrm{R}$ com pontuação $<3$ & $0,3<\mathrm{C} \leq 0,6$ & 2 \\
Pelo menos uma $\mathrm{R} \geq 3$ & $0,3<\mathrm{C} \leq 0,6$ & 3 \\
Todas as $\mathrm{R} \neq 4$ & $\mathrm{C}>0,6$ & 4 \\
Todas as $\mathrm{R}=4$ & $\mathrm{C}>0,6$ & 5 \\
\hline \multicolumn{1}{|c|}{ Graduação da severidade } & I baixo & 0 ou 1 \\
& II moderado & 2 ou 3 \\
\hline
\end{tabular}

* Pontuação (4): no original, "é muito difícil OU é impossível sem ajuda"

das, embora ele esteja sendo utilizado na literatura ${ }^{29,27-39}$. Sua grande vantagem é que mede a limitação funcional relativa à DTM, diferentemente de outros índices que avaliam especificamente a severidade dos sinais e sintomas clínicos. Assim, esse questionário apresenta-se como uma ferramenta adequada para verificar ganhos em termos funcionais após programas de tratamento ou intervenções terapêuticas.
CONCLUSÃO

Há diferentes ferramentas disponíveis na literatura para avaliação de DTM e, para que seu uso seja apropriado, a escolha da ferramenta mais adequada deve basear-se no julgamento fundamentado, vinculado ao intuito específico do pesquisador ou do clínico. Os índices consistem na verdade em roteiros de perguntas e de tópicos de avaliação organizados e pontuados, que podem fornecer a classificação da severidade da condição. Os questionários são melhor empregados em estudos epidemiológicos, para traçar perfis populacionais, para fazer triagens iniciais de pacientes. ou ainda, na avaliação de qualidade de vida. Critérios diagnósticos são apresentados no artigo de mesmo título parte II, neste número da revista. 


\section{REFERENCIAS}

1 American Society of Temporomandibular Joint Surgeons (ASTJS). Guidelines for diagnosis and management of disorders involving the temporomandibular joint and related musculoskeletal structures. Cranio. 2003;21(1):68-76.

2 Dworkin SF, Huggins KH, Leresche L, von Korff M, Howard J, Truelove E, et al. Epidemiology of signs and symptoms in temporomandibular disorders: clinical signs in cases and controls. J Am Dent Assoc. 1990;120(3):273-81.

3 Thilander B, Rubio G, Pena L, Mayorga C. Prevalence of temporomandibular disorders and its association with malocclusion in children and adolescents: an epidemiologic study related to specified stages of dental development. Angle Orthod. 2002;72(2):146-54.

4 Solow B, Tallgren A. Natural head position in standing subjects. Acta Odontol Scand. 1971;29(5):591-607.

5 Huggare J, Raustia, A. Head posture and cervicovertebral and craniofascial morphology in patients with craniomandibular dysfunction. Cranio. 1992;10(3):173-7.

6 McNeill C. Management of temporomandibular disorders: concepts and controversies. J Prosthet Dent. 1997;77(5):510-22.

7 Visscher CM, Lobbezoo F, de Boer W, van der Zaag J, Naeije M. Prevalence of cervical spinal pain in craniomandibular pain patients. Eur J Oral Sci. 2001;9(2):76-80.

8 Pedroni CR, De Oliveira AS, Guaratini MI. Prevalence study of signs and symptoms of temporomandibular disorders in university students. J Oral Rehabil. 2003;30(3):283-9.

9 Greene CS, Marbach JJ. Epidemiologic studies of mandibular dysfunction: a critical review. J Clin Prosthet Dent. 1982;48(2):184-90.

10 Rugh JD, Solberg WK. Oral health status in the United States: temporomandibular disorders. J Dent Educ. 1985;49(6):398-406.

11 Fricton JR. Clinical care for myofascial pain. Dent Clin North Am. 1991;35(1):1-28.

12 Okeson JP. Dor orofacial: guia de avaliação, diagnóstico e tratamento. São Paulo: Quintessence; 1998.

13 Helkimo M. Studies on function and dysfunction of the masticatory system, II: index for anamnestic and clinical dysfunction and occlusal state. Sven Tandlak Tidskr. 1974;67(2):101-21.
14 Fonseca DM, Bonfate G, Valle AL, Freitas SFT. Diagnóstico pela anamnese da disfunção craniomandibular. Rev Gaucha Odontol. 1994;42:23-8.

15 Fricton JR, Schiffman EL. The craniomandibular index: validity. J Prosthet Dent. 1987;58(2):222-8.

16 Pehling J, Schiffman E, Look J, Shaefer J, Lenton P, Fricton J. Interexaminer reliability and clinical validity of the temporomandibular index: a new outcome measure for temporomandibular disorders. J Orofac Pain. 2002;16(4):296-304.

17 Dworkin SF, LeResche L. Research diagnostic criteria for temporomandibular disorders: review, criteria, examinations and specifications, critique. Cranio. 1992;6(4):301-55.

18 Truelove EL, Sommers EE, LeResche L, Dworkin SF, Von Korff M. Clinical diagnostic criteria for TMD: new classification permits multiple diagnoses. J Am Dent Assoc. 1992;123(4):47-54.

19 Williamson RA, McNamara D, McAuliffe W.True eminectomy for internal derangement of the temporomandibular joint. Br J Oral Maxillofac Surg. 2000;38(5):554-60.

20 Gleissner C, Kaesser U, Dehne F, Bolten WW, Willershausen B. Temporomandibular joint function in patients with longstanding rheumatoid arthritis - I: role of periodontal status and prosthetic care; a clinical study. Eur J Med Res. 2003;27;8(3):98-108.

21 Pahkala R, Heino J. Effects of sagittal split ramus osteotomy on temporomandibular disorders in seventy-two patients. Acta Odontol Scand. 2004;62(4):238-44.

22 Hlawitschka M, Loukota R, Eckelt U. Functional and radiological results of open and closed treatment of intracapsular (diacapitular) condylar fractures of the mandible. Int J Oral Maxillofac Surg. 2005;34(6):597-604.

23 van der Weele LT, Dibbets JM. Helkimo's index: a scale or just a set of symptoms? J Oral Rehabil. 1987;14(3):229-37.

24 Gross A, Gale EM. A prevalence study of the clinical signs associated with mandibular dysfunction. J Am Dent Assoc. 1983;107:932-6.

25 Fricton JR, Schiffman EL. Reliability of a craniomandibular index. J Dent Res. 1986;65(11):1359-64.

26 Hatch JP, Rugh JD, Sakai S, Prihoda TJ. Reliability of the craniomandibular index. J Orofac Pain. 2002;16(4):284-95. 


\section{Referências (cont.)}

27 Kopp S. Reproducibility of response to a questionnaire on symptoms of masticatory dysfunction. Community Dent Oral Epidemiol. 1976;4(5):205-9.

28 Locker D, Slade G. Association of symptoms and signs of TM disorders in an adult population. Community Dent Oral Epidemiol. 1989;17(3):150-3.

29 Stegenga B, de Bont LG, de Leeuw R, Boering G. Assessment of mandibular function impairment associated with temporomandibular joint osteoarthrosis and internal derangement. J Orofac Pain. 1993;7(2):183-95.

30 Gerstner GE, Clark GT, Goulet JP. Validity of a brief questionnaire in screening asymptomatic subjects from subjects with tension-type headaches or temporomandibular disorders. Community Dent Oral Epidemiol. 1994;22(4):235-42.

31 Zulqarnain BJ, Khan N, Khattab S. Self-reported symptoms of temporomandibular dysfunction in a female university student population in Saudi Arabia. J Oral Rehabil. 1998;25(12):946-53.

32 Manfredi AS, Silva AA, Vendite LL. Avaliação da sensibilidade do questionário de triagem para dor orofacial e desordens temporomandibulares recomendado pela Academia Americana de Dor Orofacial. Rev Bras Otorrinolaringol. 2001;67(6):763-8.

33 Smith V, Williams B, Stapleford R. Rigid internal fixation and the effects on the temporomandibular joint and masticatory system: a prospective study. Am J Orthod Dentofacial Orthop. 1992;102(6):491-500.
34 Conti P, Ferreira PM, Pegoraro LF, Conti JV, Salvador MC. A cross-sectional study of prevalence and etiology of signs and symptoms of temporomandibular disorders in high school and university students. J Orofac Pain. 1996;10(3):254-62.

35 Bevilaqua-Grossi D, Chaves TC, Oliveira AS Monteiro-Pedro V. Anamnestic index severity and signs and symptoms of temporomandibular disorders (TMD). Cranio. 2006;24(2):1-7.

36 Oliveira AS, Dias EM, Contato RG, Berzin F. Prevalence study of signs and symptoms of temporomandibular disorder in Brazilian college students. Pesq Odontol Bras. 2006;20(1):3-7.

37 Holmlund AB, Axelsson S, Gynther GW. A comparison of discectomy and arthroscopic lysis and lavage for the treatment of chronic closed lock of the temporomandibular joint: a randomized outcome study. J Oral Maxillofac Surg. 2001;59(9):972-7.

38 Dijkstra PU, Stegenga B, de Bont LG, Bos RR. Function impairment and pain after closed treatment of fractures of the mandibular condyle. J Trauma. 2005;59(2):424-30.

39 De Laat A, Stappaerts K, Papy S. Counseling and physical therapy as treatment for myofascial pain of the masticatory system. J Orofac Pain. 2003;17(1):42-9. 